\title{
A flux transfer event observed at the magnetopause by the Equator-S spacecraft and in the ionosphere by the CUTLASS HF radar
}

\author{
D. A. Neudegg ${ }^{1}$, T. K. Yeoman ${ }^{1}$, S. W. H. Cowley ${ }^{1}$, G. Provan $^{1}$, G. Haerendel ${ }^{2}$, W. Baumjohann ${ }^{2}$, U. Auster ${ }^{3}$, \\ K.-H. Fornacon ${ }^{3}$, E. Georgescu ${ }^{4}$, C. J. Owen ${ }^{5}$ \\ ${ }^{1}$ Department of Physics and Astronomy, Leicester University, University Road, Leicester LE1 7RH \\ E-mail: dan2@ion.le.ac.uk \\ ${ }^{2}$ Max-Planck Institut für Extraterrestrische Physik, Garching, Munich, Germany \\ ${ }^{3}$ Technische Universität von Braunschweig, Braunschweig, Germany \\ ${ }^{4}$ ISS, Bucharest, Romania \\ ${ }^{5}$ Queen Mary and Westfield College, London, UK
}

Received: 12 November 1998 / Accepted: 20 November 1998

\begin{abstract}
Observations of a flux transfer event (FTE) have been made simultaneously by the Equator-S spacecraft near the dayside magnetopause whilst corresponding transient plasma flows were seen in the nearconjugate polar ionosphere by the CUTLASS Finland $\mathrm{HF}$ radar. Prior to the occurrence of the FTE, the magnetometer on the WIND spacecraft $\sim 226 \mathrm{R}_{\mathrm{E}}$ upstream of the Earth in the solar wind detected a southward turning of the interplanetary magnetic field (IMF) which is estimated to have reached the subsolar magnetopause $\sim 77 \mathrm{~min}$ later. Shortly afterwards the Equator-S magnetometer observed a typical bipolar FTE signature in the magnetic field component normal to the magnetopause, just inside the magnetosphere. Almost simultaneously the CUTLASS Finland radar observed a strong transient flow in the $\mathrm{F}$ region plasma between $78^{\circ}$ and $83^{\circ}$ magnetic latitude, near the ionospheric region predicted to map along geomagnetic field lines to the spacecraft. The flow signature (and the data set as a whole) is found to be fully consistent with the view that the FTE was formed by a burst of magnetopause reconnection.
\end{abstract}

Key words. Interplanetary physics (ionospheremagnetosphere interaction) Magnetospheric physics (magnetopause, cusp, and boundary layers; solar windmagnetosphere interactions)

\section{Introduction}

Transient signatures termed "flux transfer event" (FTEs) were first observed at the Earth's dayside magnetopause by Russell and Elphic (1978) in magnetic

Correspondence to: D. A. Neudegg field data from the ISEE-1 and -2 spacecraft, and by Haerendel et al. (1978) in equivalent data from HEOS-2. The principal effects consist of a few-minute bipolar excursion of the field component normal to the magnetopause, together with evidence of mixed magnetosheath-magnetosphere plasma populations. Subsequent work as shown a statistical association with southward fields in the IMF (e.g. Rijnbeek et al., 1984). Although most evidence points to pulsed reconnection as the source of FTEs (e.g. Lockwood, 1991), it has also been suggested that they may result from changes in the plasma pressure external to the magnetosphere (e.g. Sibeck, 1994).

In the 20 years since their discovery there has been considerable interest in the ionospheric signature of FTEs, since these provide means of examining their "global" characteristics, and also allow discrimination between models of their physical origins. If we consider the ionospheric flow, in particular, then pulsed reconnection should result in the formation of "flow channels" on newly-opened cusp field lines of 5-10 min duration, where the flow direction depends on the direction of the IMF (Cowley and Lockwood, 1992). Pressure changes should instead produce paired flow vortices mainly on closed field lines, which should propagate azimuthally away from noon (Kivelson and Southwood, 1991). Both types of flow have been reported in the literature, the former e.g. by Lockwood et al. (1990), Pinnock et al. (1993, 1995), Milan et al. (1998) and Provan et al. (1998), and the latter e.g. by Glassmeier (1992), and Lühr et al. (1993). However, there have been very few opportunities to date to examine directly the relationship between FTEs observed at the magnetopause and their ionospheric flow signatures. In fact only one published observation is known to us, by Elphic et al. (1990). Although the radar flow data employed by these authors were of limited spatial coverage and temporal resolution (two radar pointing directions with a 5 min cycle), they were sufficient to provide one example of the simultaneous occurrence of FTEs at the magnetopause and pulsed 
ionospheric flows of similar recurrence time. This work addresses two questions. First, is it possible to demonstrate a convincing connection between individual FTEs observed at the magnetopause and corresponding transient flows in the ionosphere? Second, if this is the case, can the flow observations be used to provide information about the physical origins of the FTE? Here we discuss an interval of simultaneous Equator-S magnetic field data from the vicinity of the magnetopause, highly space- and time-resolved flow data from the CUTLASS Finland radar, and contextual information on the upstream interplanetary medium from the WIND spacecraft, to show that we can answer "yes" to both these questions.

\section{Instrumentation}

\subsection{Interplanetary data: WIND spacecraft}

Data on the interplanetary field and plasma conditions upstream of the magnetosphere during the interval $\sim 0900-1000$ UT on 4 January 1998 were obtained by the magnetometer (Lepping et al., 1995) and 3DP plasma instrument (Lin et al., 1995) on the WIND spacecraft. During this interval the spacecraft was located at GSM $(X, Y, Z)=(226,21,-3) \mathrm{R}_{\mathrm{E}}$. Using prevailing interplanetary parameters (a solar wind speed and density of $\sim 345 \mathrm{~km} \mathrm{~s}^{-1}$ and $\sim 6 \mathrm{~cm}^{-3}$ ), the delay for IMF features to propagate from the spacecraft to the subsolar magnetopause is estimated to have been $\sim 77 \pm 5$ min. The slowing of the flow across the bow shock and in the magnetosheath has been taken into account in making this estimate, where we have used the empirical bow shock and magnetopause models of Peredo et al. (1995) and Roelof and Sibeck (1993) (hereafter referred to as $\mathrm{R} \& S)$.

\subsection{Magnetopause: Equator-S spacecraft}

The Equator-S spacecraft was launched on 2 December, 1997, into an initial geostationary transfer orbit, and was then boosted to a higher apogee on 11 December. The FTE described here was observed near apogee at $\sim 1020$ UT ( 0910 MLT) on 4 January, 1998, when the spacecraft was $\sim 11.3 \quad \mathrm{R}_{\mathrm{E}}$ from Earth at GSM $(X, Y, Z)=(8,7,4) \mathrm{R}_{\mathrm{E}}$. This occurred during the spacecraft commissioning phase when only the magnetometer was continuously operating. The spacecraft trajectory is shown in Fig. 1a, together with two R\&S magnetopause positions corresponding to interplanetary conditions appropriate to the interval (see later).

\subsection{Polar ionosphere: CUTLASS Finland HF radar}

Details of the CUTLASS HF radars are given in Milan et al. (1997). The CUTLASS Finland radar field-of-view (FOV) is shown in Fig. 1b, where ionospheric plasma velocities are displayed for 1022 UT on 4 January, 1998, (discussed later). During this experiment a special high space- and time-resolution scan mode was run, with $30 \mathrm{~km}$ range gates and with each beam scanned for $2 \mathrm{~s}$ in a $32 \mathrm{~s}$ sweep over the 16 beams (from beam 15 on the east of the FOV to beam 0 on the west). Detailed data from beam 5 (highlighted in Fig. 1b) will be presented below. The geomagnetic field lines from Equator-S have been mapped to the ionosphere using the Tsyganenko 89 a model for the actual $K p$ value of 0 . This model predicts the spacecraft to have been on open field lines during the interval 0900-1300 UT, whereas the magnetometer data show that Equator-S was located on magnetospheric field lines around the time of the FTE observation at $\sim 1020$ UT. It has therefore been assumed that the continuation of the ground track from 0900 UT along a smooth arc (dashed in Fig. 1b) will place the footprint of Equator-S during the FTE observation near the far ranges of the radar adjacent to beam 0 .

\section{Results}

The observations are shown in Fig. 2, where the Equator-S field and CUTLASS velocity data span the interval 1000-1100 UT, while the WIND magnetic field and dynamic pressure data have been shifted by the estimated propagation delay of $77 \mathrm{~min}$ to the subsolar magnetopause. The top three panels show the IMF components in GSM coordinates measured by the WIND spacecraft over the interval 0843-0943 UT. At the start of the interval $B_{z}$ was northward (and had been so nearly continuously for more than the previous hour), $B_{y}$ was positive, and $B_{x}$ was near zero. At 0851 UT (arrowed, corresponding to 1008 UT at the subsolar magnetopause) $B_{z}$ turned consistently but weakly southward $(\sim-0.5 \mathrm{nT})$, followed by stronger southward fields ( -2 to $-3 \mathrm{nT}$ ) at $0903 \mathrm{UT}$ (also arrowed, $\sim 1020$ UT at the subsolar magnetopause). Shortly before the latter increase, at 0901 UT, a $25 \%$ increase occurred in the solar wind dynamic pressure, shown in the fourth panel, from 1.4 to $1.8 \mathrm{nPa}$. The IMF then returned to a northward configuration at 0925 UT ( 1042 UT at the subsolar magnetopause).

The model magnetopause shown by the solid line in Fig. 1a corresponds to interplanetary conditions typical of the start of the interval in Fig. 2 (dynamic pressure $P=1.4 \mathrm{nPa}$ and $B_{z}=1 \mathrm{nT}$ ), while that shown by the dashed line corresponds to conditions after the southward turn of the IMF and the pressure increase $\left(P=1.8 \mathrm{nPa}\right.$ and $\left.B_{z}=-3 \mathrm{nT}\right)$. The spacecraft at apogee is thus expected initially to lie inside the magnetopause, whilst the combined action of the flux erosion associated with the southward IMF and the increased pressure is expected to bring the boundary close to the spacecraft. Flux erosion is the dominant effect according to the Roelof and Sibeck (1993), model, reducing magnetopause distance by $1.5 \mathrm{R}_{\mathrm{E}}$ compared with $0.5 \mathrm{R}_{\mathrm{E}}$ predicted for the pressure increase. The centre three panels of Fig. 2 then show the Equator-S magnetometer data in boundary normal (LMN) coordinates, defined by using a $\mathrm{R} \& \mathrm{~S}$ magnetopause typical of the interval as 


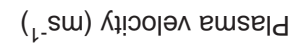

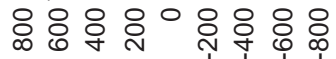
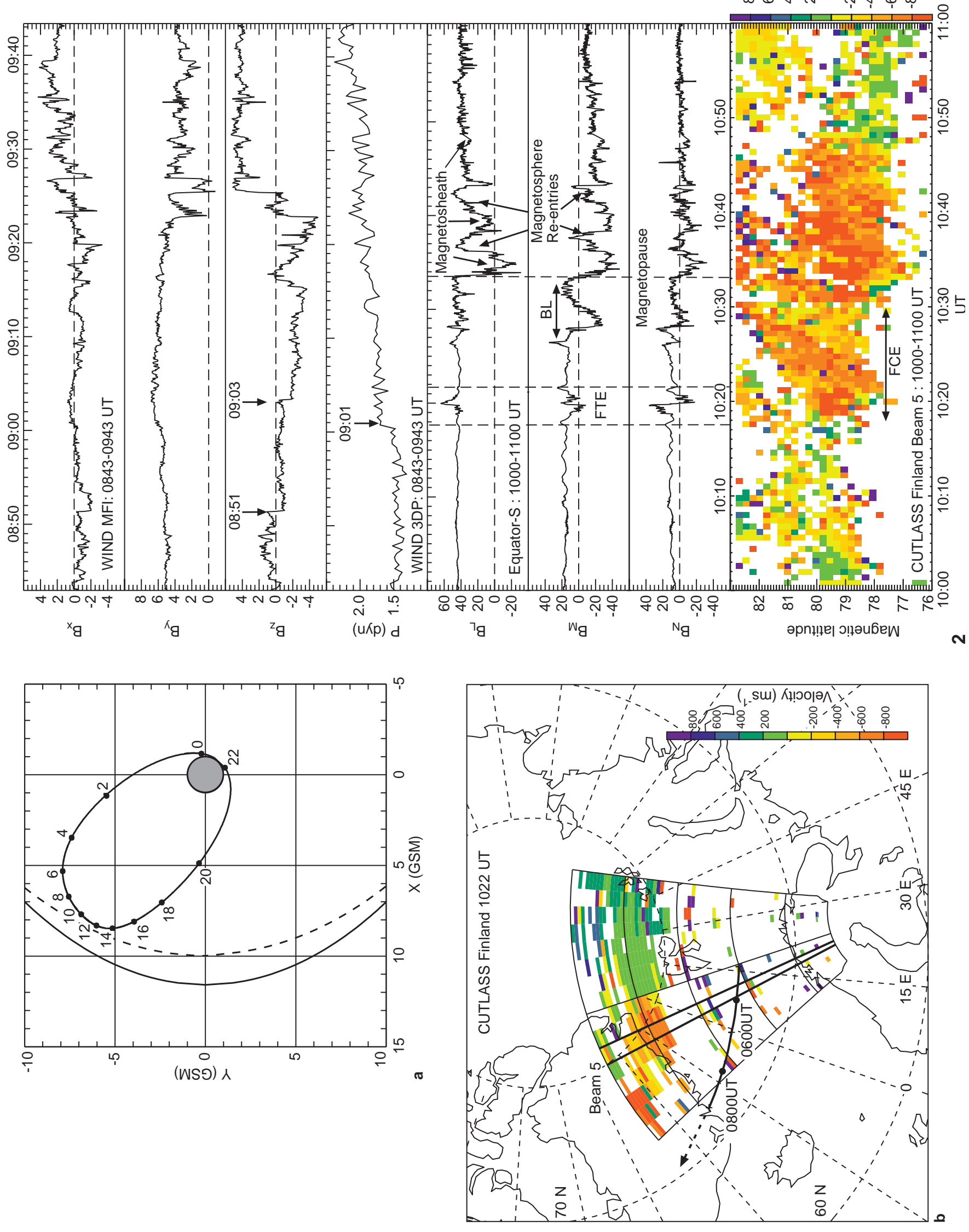
Fig. 1a The Equator-S orbit for 4 January 1998 in the $X$ - $Y$ GSM plane. The dots indicate the spacecraft position at various UT hours. Two Roelof and Sibeck (1993) model magnetopauses are also shown (see text for details). b The field-of-view of the CUTLASS Finland radar showing ionospheric plasma velocity measurements for the $32 \mathrm{~s}$ scan starting at 1021:43 UT on 4 January, 1998, at a sounding frequency of $10 \mathrm{MHz}$. Beam 5 is highlighted, with beam 0 located on the western edge of the FOV, and beam 15 on the eastern edge. The location of Equator-S for the UT times shown has been mapped along model magnetic field lines to the FOV (solid line)

Fig. 2. From top to bottom we show; interplanetary magnetic field (nT - GSM coordinates) and solar wind dynamic pressure (nPa) data from WIND; magnetic field (nT - boundary normal coordinates) at the Equator-S spacecraft; the line-of-sight velocity data from beam 5 of the CUTLASS Finland HF radar, for the interval 10-11 UT on 4 January 1998. The WIND data has been shifted by the estimated 77 minute propagation delay from the spacecraft to the subsolar magnetopause

a whole $\left(P=1.25 \mathrm{nPa}\right.$ and $\left.B_{z}=0 \mathrm{nT}\right)$, though the results are not sensitive to the exact choice. $N$ is the component normal to the magnetopause, positive outwards, $L$ is tangential to the magnetopause so that the $N$ - $L$ plane contains the $Z$ GSM axis, and $M$ is the orthogonal westward component. For comparison with the IMF data, $L$ is similar to $Z$ GSM, $M$ to $-Y$, and $N$ is a combination of $X$ and $Y$ due to the pre-noon location of the spacecraft. As expected from the above, at the start of the interval Equator-S was in the magnetosphere, as indicated by a relatively steady field in the $+L$ direction. A "classic" bipolar magnetospheric FTE was then observed in the $\mathrm{N}$ component, starting at $1018 \mathrm{UT}$, indicated by the vertical dashed lines. This signature is the primary focus of the present work. If we assume that the FTE resulted from a burst of reconnection in the subsolar region, the propagation delay to the spacecraft would have been just a few $(\sim 5)$ minutes. In this case the FTE would have been formed shortly $(\sim 5 \mathrm{~min})$ after the magnetosheath field turned weakly southward at the subsolar magnetopause. We note, however, that the FTE also occurred in approximate coincidence with the estimated arrival of the increased solar wind dynamic pressure mentioned above, which was observed by WIND at $\sim 0901$ UT. At $\sim 1028$ UT the spacecraft began a 6-min transit through the presumed magnetopause boundary layer (BL), characterised by disturbed field components indicative of the presence of higher- $\beta$ plasma. The $M$ component was also deflected negative on entry, towards the direction of the magnetosheath field outside. These observations are indicative of a subsequent interval of more continuous magnetopause reconnection, though perhaps with significant variations superposed, as indicated by the FTE-like bipolar $N$ component signatures observed during entry to the layer (1026-1030 UT). A magnetopause crossing (MP) then occurred at 1034 UT, where all the field components changed sense to match the IMF polarity outside. Subsequently, there were two more probable re-entries to BL-like fields centred on 1037 and 1042 UT as marked in the figure, suggesting that reconnection may still have been in progress until $\sim 1043$ UT. This was then followed by an excursion into a northward magnetosheath field to the end of the interval.
The CUTLASS Finland radar data shown in the bottom panel of Fig. 2 are taken from beam 5, which points towards the invariant geomagnetic pole, although it is oriented $5^{\circ}-10^{\circ}$ westward of the normal to the local $L$-shells (Fig. 1b). Negative velocities (yellow and red) indicate flow away from the radar. At the beginning of the interval the flows were generally below $200 \mathrm{~m} \mathrm{~s}^{-1}$ with variable directions. At 1018 UT, in coincidence with the FTE, a transient 'flow channel event' (FCE) began near $79^{\circ}$ magnetic latitude with velocities $400-800 \mathrm{~m} \mathrm{~s}^{-1}$ away from the radar along the line-ofsight. Simultaneously, the equatorward border of the region of backscatter moved equatorward, indicative of the erosion of closed flux if the region of backscatter is related to the region of cusp precipitation (Milan et al., 1998). Examination of the flow observed in all the radar beams, such as those in the scan at 1022 UT shown in Fig. 1b, indicates that the enhanced flow was predominantly westward with a speed of $1000 \pm 200 \mathrm{~m} \mathrm{~s}^{-1}$. Subsequently, the region of high speed flow moved poleward, reaching $82^{\circ}$ after $10 \mathrm{~min}$. The equatorward border of the backscatter also recovered poleward during this interval. Overall, this behaviour is entirely typical of the class of dayside transient phenomena studied e.g. by Pinnock et al. (1993, 1995), Milan et al. (1998) and Provan et al. (1998), and attributed to the ionospheric signature of transient reconnection. Subsequently, as these flows died away at $\sim 1030$ UT at $\sim 82^{\circ}$, a new onset of enhanced flow commenced at $78^{\circ}$, with westward velocities peaking at $1500 \pm 500 \mathrm{~m} \mathrm{~s}^{-1}$. This onset occurred $\sim 2$ min after the spacecraft entered the BL. The equatorward border of the region of backscatter again moved equatorward at the onset of flow, and later only partially recovered, consistent with an overall expansion of the polar cap. Finally, the field in the magnetosheath appears to have turned northward at $\sim 1043$ UT, in agreement with the time-shifted IMF values. The enhanced ionospheric flow then died away, reaching values similar to those at the start of the interval by $\sim 1049$ UT.

\section{Discussion and summary}

In this study we have presented the most detailed observations to date of the nearly simultaneous occurrence of an FTE at the dayside magnetopause and transient flows in the near-conjugate ionosphere. These signatures were observed a few minutes after a southward turn of the IMF is estimated to have arrived at the subsolar magnetopause, and follow a previous interval of northward IMF in which no such features were observed either at the spacecraft or by the radar. This context strengthens the case that these events were causally linked, and have their origin in a pulse of reconnection at the magnetopause. However, they also occurred in approximate coincidence with the estimated arrival of a modest increase in the solar wind dynamic pressure, such that it is important to examine this as a possible cause of the field and flow transients. The radar data show that the transient effects in the ionosphere 
comprised consistently westward-directed flows as expected from the tension effect of the $+Y$ field component in the IMF, together with an initial equatorward motion of the region of backscatter indicative of an equatorward motion of the cusp and the opening of closed flux. Subsequently the region of enhanced westward flow and the equatorward border of the backscatter both migrated polewards over an interval of $\sim 10$ min. These characteristics are fully consistent with those expected for a pulse of enhanced reconnection at the magnetopause (Cowley and Lockwood, 1992). No evidence was found for the azimuthally propagating flow reversals which would be indicative of a travelling twin-vortex, as would be expected if the transients were formed directly as a result of the pressure change. The data set as a whole is thus fully consistent with the view that the FTE was formed by a burst of reconnection which occurred shortly after the southward turn of the IMF.

Considering the relative timing of the space and ionospheric events, the near-simultaneous appearance of the FTE at the magnetopause and the transient westward flow channel in the conjugate ionosphere is compatible with a few-minute propagation of the FTE from an original reconnection site (e.g. in the subsolar region) to the spacecraft, together with a few-minute propagation of the field tension effect along the newly opened field lines to the ionosphere as an Alfvén wave, and from thence into the radar FOV. However, other events with different source points may have different few-minute relative delays depending upon the propagation paths from the point of origin to the points of observation. The second longer ( $\sim 18 \mathrm{~min})$ interval of enhanced ionospheric flow was initiated $\sim 2$ min after Equator-S entered the BL, and was probably associated with a more enduring interval of magnetopause reconnection during which the spacecraft passed through the BL and MP (probably several times), and entered the magnetosheath. This flow died away $\sim 5$ min after the magnetosheath field returned to a northward orientation, again consistent with a reconnection interpretation.

Acknowledgements. We thank the Equator-S staff at Max-Planck Institut für Extraterrestrische Physik (MPE), the German Space Operations Centre (GSOC) and TU Braunschweig, the University of Leicester CUTLASS HF radar team, S. E. Milan for SuperDARN radar data visualisation software, NASA/GSFC (T. Perry and R. Lepping) for WIND MFI magnetometer data, U. Berkeley (S. Bale and R. Lin) for WIND 3DP data and SSCWeb for orbit predictions and magnetic field mappings. This project was made possible through grant 50 0C 94024 of the German Space Agency (DARA). D. A. Neudegg was supported by PPARC grant GR/ L29767, and G. Provan by PPARC grant PPA/G/O/1997/00254.

Topical Editor D. Alcaydé thanks three referees for their help in evaluating this paper.

\section{References}

Cowley, S. W. H., and M. Lockwood, Excitation and decay of solar wind-driven flows in the magnetosphere-ionosphere system, Ann. Geophysicae, 10, 103-115, 1992.
Elphic, R. C., M. Lockwood, S. W. H. Cowley, and P. E. Sandholt, Flux transfer events at the magnetopause and in the ionosphere, Geophys. Res. Lett., 17, 2241-2244, 1990.

Glassmeier, K.-H., Traveling magnetospheric convection twinvortices: observations and theory, Ann. Geophysicae, 10, 547$565,1992$.

Haerendel G., G. Paschmann, N. Sckopke, H. Rosenbauer, and P. C. Hedgecock, The frontside boundary layer of the magnetosphere and the problem of reconnection, J. Geophys. Res., 83, 3195-3216, 1978.

Kivelson, M. G., and D. J. Southwood, Ionospheric travelling vortex generation by solar wind buffeting of the magnetosphere, J. Geophys. Res., 96, 1661-1667, 1991.

Lepping, R. P. et al., The WIND magnetic field investigation, Space Sci. Rev., 71, 207-229, 1995.

Lin R. P., K. A. Anderson, S. Ashford, C. Carlson, D. Curtis, R. Ergun, D Larson, J. McFadden, M. McCarthy, G. K. Parks, H. Réme, J. M. Bosqued, J. Coutelier, F. Cotin, C. D’Uston, K.-P. Wenzel, T. R. Sanderson, J. Henrion, J. C. Ronnet and G. Paschmann, A three dimensional plasma and energetic particle investigation for the WIND spacecraft, Space Sci. Rev., 71, 125-153, 1995.

Lockwood, M., Flux transfer events at the dayside magnetopause: transient reconnection or magnetosheath pressure pulses?, J. Geophys. Res., 96, 5497-5509, 1991.

Lockwood, M., P. E. Sandholt, A. D. Farmer, S. W. H. Cowley, B. Lybekk, and V. N. Davda, Auroral and plasma flow transients at magnetic noon, Planet. Space Sci., 38, 973-993, 1990.

Lühr, H., W. Blawert, and H. Todd, The ionospheric plasma flow and current patterns of travelling convection vortices: a case study, J. Atmos. Terr. Phys., 55, 1717-1727, 1993.

Milan, S. E., T. K. Yeoman, M. Lester, E. C. Thomas, and T. B. Jones, Initial backscatter occurrence statistics from the CUTLASS HF radars, Ann. Geophysicae, 15, 703-718, 1997.

Milan, S. E., M. Lester, S. W. H. Cowley, J. Moen, P. E. Sandholt, and C. J. Owen, Meridian-scanning photometer, coherent HF radar and magnetometer observations of the cusp: a case study, Ann. Geophysicae, in press, 1998.

Peredo, M., J. A. Slavin, E. Mazur, and S. A. Curtis, Threedimensional position and shape of the bow shock and their variation with Alfvénic, sonic and magnetosonic Mach numbers and interplanetary magnetic field orientation, J. Geophys. Res., 100, 7907-7916, 1995.

Pinnock, M., A. S. Rodger, J. R. Dudeney, K. B. Baker, R. A. Greenwald, and M. Greenspan, Observations of an enhanced convection channel in the cusp ionosphere, J. Geophys. Res., 98, 3767-3776, 1993.

Pinnock, M., A. S. Rodger, J. R. Dudeney, F. Rich, and K. B. Baker, High spatial and temporal resolution observations of the ionospheric cusp, Ann. Geophysicae, 13, 919-925, 1995.

Provan, G., T. K. Yeoman, and S. E. Milan, CUTLASS Finland radar observations of the ionospheric signatures of flux transfer events and the resulting plasma flows, Ann. Geophysicae., in press, 1998.

Rijnbeek, R. P., S. W. H. Cowley, D. J. Southwood, and C. T. Russell, A survey of dayside flux transfer events observed by the ISEE-1 and -2 magnetometers, J. Geophys. Res., 89, 786-800, 1984.

Roelof, E. C., and D. G. Sibeck, Magnetopause shape as a bivariate function of interplanetary magnetic field $B z$ and solar wind dynamic pressure, J. Geophys. Res., 98, 21421-21450, 1993.

Russell, C. T., and R. C. Elphic, Initial ISEE magnetometer results: magnetopause observations, Space Sci. Rev., 22, 681-715, 1978.

Sibeck, D. G., Magnetosheath magnetic field variability, $A d v$. Space Res., 14, (7)91-(7)94, 1994. 